\title{
Research on Optimization of Financial Sharing Model Based on Blockchain Technology
}

\author{
Dong Cheng*, Yu Cheng
}

School of Accounting, Anhui University of Finance and Economics, Bengbu 233030, Anhui, China. E-mail: cd19991222@126.com

\begin{abstract}
The financial sharing model has gradually developed with the rise of group companies, but in the process of development, many problems such as unreasonable overall structure and centralized power have emerged. As a brand-new accounting information application technology, blockchain technology has the characteristics of distributed, decentralized, anti-counterfeiting and traceability, and it can be widely used in many fields. This article starts from the basic architecture of the blockchain, establishes an infrastructure model for the financial sharing model and a specific optimization path, and it finally designs an internal and external "dual chain" structure based on the financial sharing model under the blockchain technology, hoping to be a financial sharing model. The current development dilemma is looking for a new way out.
\end{abstract}

Keywords: Financial Sharing; Blockchain; System Design

\section{Introduction}

The information technology in the Internet era provides technical support for the realization of financial reforms. As a new management model that improves efficiency and meets the development needs of enterprises, the financial shared service model has gradually developed with the rise of group companies, but it is still developing. In the process, many problems such as unreasonable total score structure and centralization of power emerged. In the continuous exploration in recent years, many scholars have proposed a new development direction of "blockchain + financial sharing”. Based on blockchain technology, it can realize a privacy protection, a safe storage and anti-tampering services of business activity data.

In recent years, scholars have conducted multi-level explorations on the "blockchain + financial sharing" model. Ying He believes that strategy, organization, process, personnel, technology and information systems are the key factors of group financial cloud computing ${ }^{[1]}$. Chunfang Zhang pointed out that the construction of a corporate financial sharing center is inseparable from strict control of corporate processes and effective integration of resources ${ }^{[2]}$. Based on the existing problems of Haier Group's financial sharing services at this stage, Jing Xie proposed that the construction of a financial sharing center should pay attention to dimensions such as organizational structure, business processes, and employee management ${ }^{[3]}$. Jianming Zhu, Yibo Hao and Biao Song conducted a performance analysis of the financial sharing system based on the alliance blockchain ${ }^{[4]}$. Limei Wu et al. conducted that the construction of financial sharing services is reflected in expense reimbursement management, centralized accounting, and centralized fund payment ${ }^{[5]}$.

In summary, the financial sharing model based on blockchain technology is still in a state of theoretical research,

Copyright (C) 2020 Dong Cheng et al.

doi: $10.18686 /$ aat.v2i3.1338

This is an open-access article distributed under the terms of the Creative Commons Attribution Non-Commercial License

(http://creativecommons.org/licenses/by-nc/4.0/), which permits unrestricted non-commercial use, distribution, and reproduction in any medium,

provided the original work is properly cited. 
and there is still a lack of thinking about how to improve and implement it. Starting from the basic structure of the blockchain, this article will construct the internal and external "dual-chain" blockchain architecture, internally dividing the financial sharing into a strategic module, a financial module, and a capital module. It will establish a financial sharing center alliance chain, and build between external parent and subsidiary companies. Subsidiary-Sharing Center Alliance Chain and the overall framework are designed based on the blockchain thinking financial sharing model accordingly.

\section{Financial sharing and blockchain}

\subsection{Introduction to financial sharing model}

The financial sharing model is generally applied to enterprise group companies or large companies. Its core is to establish a corporate centralized management model, which concentrates the financial personnel and standardized financial accounting services of each unit of the group company into a new cost center or profit center. In the financial information system, it provides professional services for different units in the enterprise to improve management efficiency. The main idea of the financial sharing center is "professional stratification and business integration", which is divided into three parts: strategy module, financial module, and capital module. Each subsidiary uploads the company's internal financial information to the financial sharing center, and the financial sharing center makes unified decisions to enhance the value of finance for strategic decision-making and business support, and eliminate industry-financial barriers.

\subsection{Insufficiency of financial sharing}

Many large companies in China established financial shared service centers ten years ago with a clear development path, and completed the construction of financial shared service centers long ago. But with the implementation of the financial sharing model, its potential problems have gradually emerged. Cuixia Jin took the group as an example and proposed that the financial sharing model needs to be strengthened in personnel training and operation management ${ }^{[6]}$. Yongmei Zhang et al. believes that the traditional financial sharing model has stagnated human development, because the organization is becoming bloated, and data security is threatened ${ }^{[7]}$. According to Haier's data, about $70 \%$ of financial personnel are engaged in strategic and business finance, and basic financial personnel and professional financial personnel account for only 30\%. The structure tends to be centralized, and it is difficult to achieve efficient implementation strategies and financial sharing centers. More and more seriously, it restricts the long-term development of enterprises.

\subsection{Analysis of the advantages of blockchain + financial sharing}

Blockchain technology is an innovative application of a new generation of information technology that uses unique nodes for distributed storage, verification, and information transmission and exchange of network data information. It has the advantages of decentralization, complete disclosure, information cannot be tampered with, and traceability. Combining blockchain technology with the financial sharing model can improve the problems of bloated organization, data trust, and internal control under the traditional financial sharing model.

\section{Research on optimization of financial sharing model based on block- chain technology}

\subsection{Infrastructure model for optimization research of financial sharing mode based on block- chain technology}

Starting from the underlying architecture of the blockchain, this article explores the underlying architecture model of the financial sharing model based on blockchain technology, which is mainly divided into six layers. 


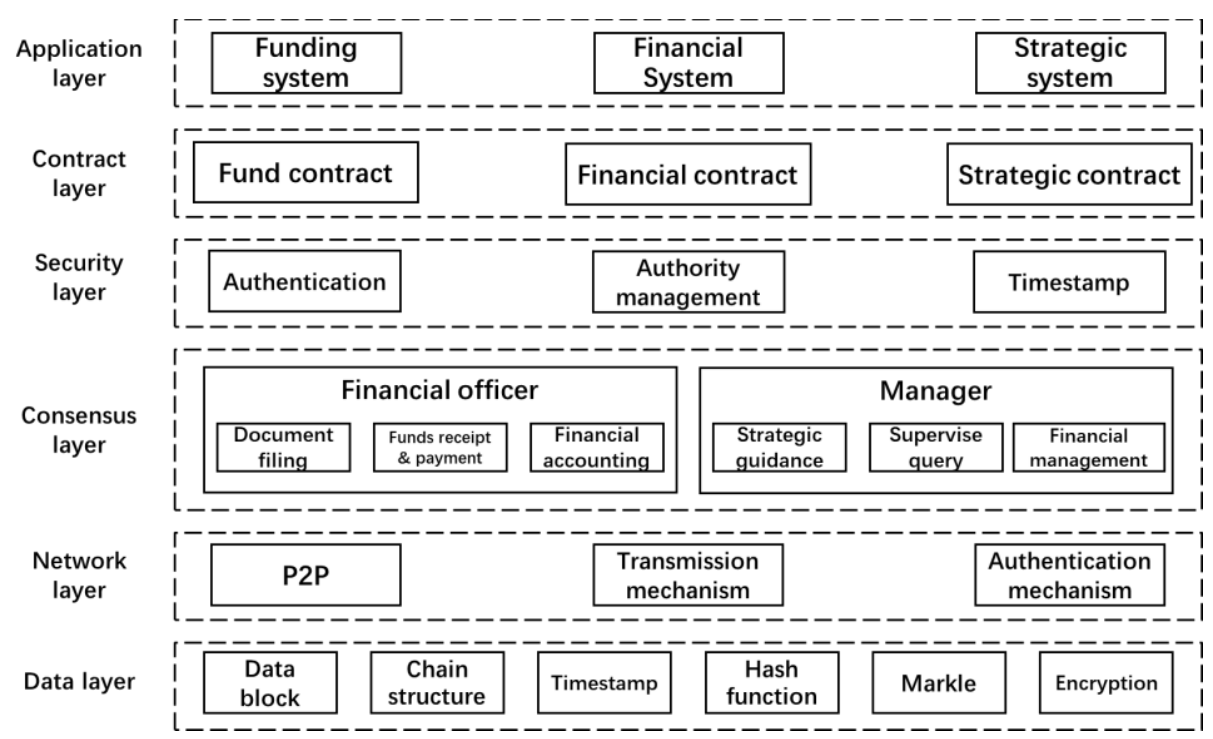

Figure 1. The basic architecture model of the optimization research of financial sharing mode based on blockchain technology.

Data layer: The data layer of the model mainly covers data algorithms. It provides the feasibility of blockchain technology operation and is also the basic layer of the blockchain structure. It mainly includes data blocks, chain structures, timestamps, hash functions, Markle trees and Asymmetric encryption technology.

Network layer: The network layer of the model mainly transmits information through the P2P network. Its main feature is that it has changed from a centralized structure to a distributed structure, which greatly reduces the degree of centralization.

Consensus layer: The consensus layer of the model is mainly embodied in the consensus mechanism, which realizes the synchronization and consistency of the data records of all nodes on the blockchain, and ensures the transparency and data sharing of the blockchain system. The model divides the financial sharing consensus mechanism into two parts for financial personnel and management personnel. Due to the complicated internal accounts of the group, the basic business of financial personnel can be transparent through the consensus mechanism, and the management consensus mechanism can strengthen internal supervision and management.

Security layer: The security layer provides security guarantees for the entire model, ensuring information security supervision through identity verification, authority management, and time stamp functions.

Contract layer: The contract layer introduces the concept of smart contracts in blockchain technology, which helps the entire financial system to manage intelligently. With the assistance of the security layer, the contract layer automatically completes data processing under inspection.

Application layer: The application layer of this model is mainly embodied in the capital system, financial system, and strategic system.

\subsection{The specific optimization path of the research on the optimization of the financial sharing mode based on blockchain technology}

The financial sharing model mainly has three functions: financial management, capital management, and strategic management. This article optimizes its basic business based on blockchain thinking, as shown in Figure 2. 


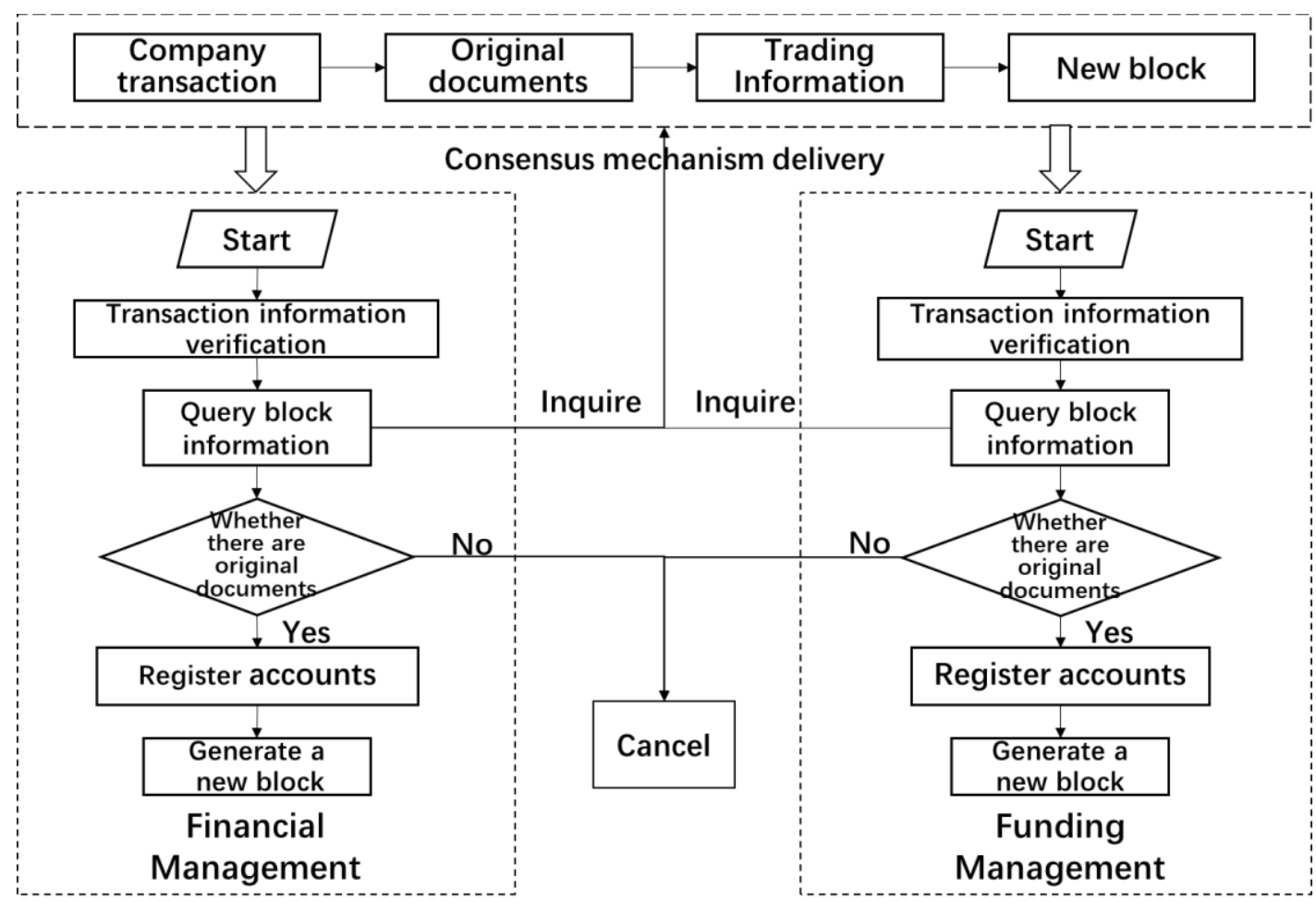

Figure 2. Basic business under blockchain thinking.

In the first step, blockchain technology packs each record into block data, which is spread through the network layer. The application of the consensus mechanism helps the data to achieve openness, that is, every record will be published in the financial sharing system, and the information is open and transparent.

In the second step, the financial system and capital system under this model are synchronized. By citing smart contract technology and identification mechanisms, the financial system and capital system automatically register accounting activities by querying whether there is true transaction information in the block information. Children pay (receive) cash.

In the third step, the financial system and capital system will generate a new block from this operation record and publish it in the blockchain financial sharing network.

\subsection{The overall design framework of the optimization research on the financial sharing mod- el based on blockchain technology}

Combined with the introduction of the previous article, this article designs the overall design framework for the optimization of the financial sharing model based on blockchain technology as shown in Figure 3. The overall idea is presented as follows.

Step 1: The transaction information of each subsidiary is entered into the blockchain platform and published to the blockchain platform through the P2P broadcast mechanism. The information recorded on the chain is determined and cannot be tampered with, and it is encrypted using cryptographic principles. With strict control of access rights, information security can be guaranteed at a higher level.

Step 2: A financial blockchain, a capital blockchain and a strategic blockchain are established based on the original financial system, capital system and strategic system of the financial sharing center, and the three will form a sharing center blockchain alliance. The financial blockchain and the capital blockchain support each other and provide feedback, and at the same time they provide data decision-making for the strategic blockchain, and the strategic blockchain is the highest authority of the financial sharing center while supervising the financial blockchain and the capital blockchain implementation. 


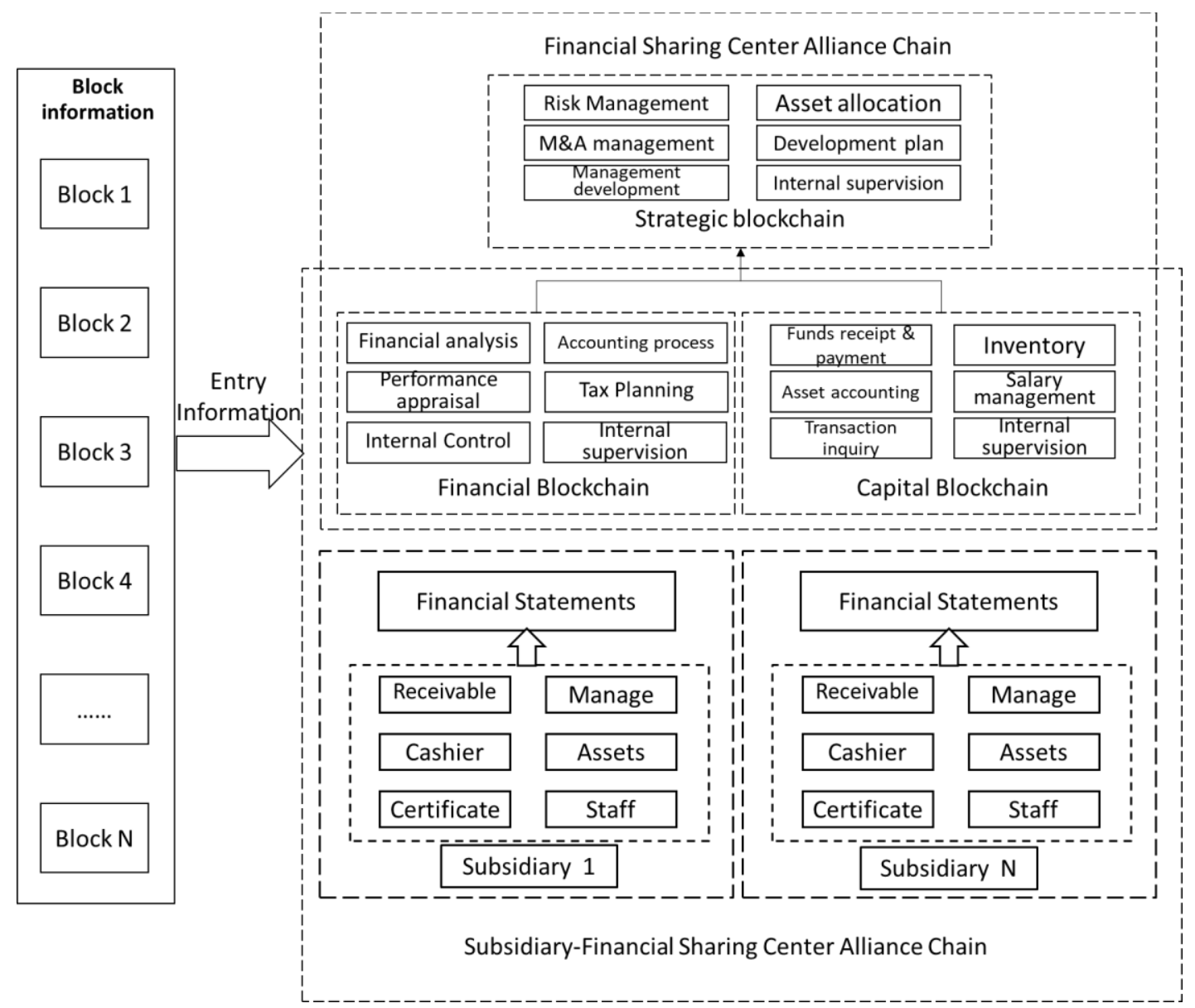

Figure 3. The overall design framework of the optimization research on the financial sharing model based on blockchain technology.

Step 3: A subsidiary-sharing center alliance chain is established based on the information provided by the original subsidiary for the financial sharing center, that is, the transaction information of each subsidiary is transparent and open. The accounting system and capital system of the subsidiary are supervised and controlled by the corresponding block chain in the financial sharing center. The capital operation activities carried out by the company do not require any third-party participation, which can save commission fees and reduce the cost of capital allocation. The supervision and management of the financial system have also increased trust in financial management.

\section{Conclusion}

With the development of the Internet era, the financial sharing model is reaching new heights. The blockchain + financial sharing model solves the problems of over-centralization and low security under the traditional financial sharing model, and also provides a guarantee for the implementation of corporate group strategies. Through the application of blockchain technology, core information such as corporate transactions can become more open and transparent, and the financial sharing center will make unified decisions, enhance the value of financial support for strategic decisions and business support, and eliminate the gap between business and finance. This article establishes a specific model for financial sharing, hoping to provide reference value for the future research and development of financial sharing model.

\section{Acknowledgement}

This article is the research results of Anhui University of Finance and Economics undergraduate research and innovation fund project, project number: XSKY20106 


\section{References}

1. He Y. The new trend of corporate financial process reengineering: Financial shared services (in Chinese). Communication of Finance and Accounting 2010; (2): 110-113.

2. Zhang C. Analysis on the construction of financial sharing center in enterprise management (in Chinese). Modern Economic Information 2017; (24): 33-34.

3. Xie J. The optimization research of Haier financial shared service [MSc thesis]. Shanghai: Donghua University; 2018.

4. Zhu J, Hao Y, Song B. Financial sharing model based on blockchain and its benefit analysis (in Chinese). Economic Issues 2019; (10): 113-120.

5. Wu L, Ding J, Wang S, et al. Financial sharing model architecture based on blockchain technology (in Chinese). Friends of Accounting 2019; (2): 149-154.

6. Jin C. Research on the evaluation system of financial shared service model of A Group (in Chinese) [MSc thesis]. Guilin: Guilin University of Electronic Technology; 2019.

7. Zhang Y, Xu Z. Research on the application of blockchain technology in financial shared services (in Chinese). Aeronautical Finance and Accounting 2020; 2(1): 16-23. 